Sollte vielleicht vorwaltendes ätzendes Alkali die $0 x y-$ dation befördern? oder die arsenige Säure in verschiedenen Zuständen existiren? Auf die Anwendbarkeit der Methode würde es in keinem Falle von Nachtheil gewesen sein. Man hätte nämlich nach der. Lösung der arsenigen Säure in kohlensaurem Natron die Flüssigkeit mit verdünnter Schwefelsäure ansäuern, dann bis zum Liter verdünnen, und im Augenblick des Gebrauchs mit kohlensaurem Natron übersättigen müssen. Im sauren Zustande kann die arsenige Säure in keinem Falle Sauerstoff aufnehmen. Allein nach meinen obigen Beobachtungen fand ich diels nicht nothwendig. Die einzige Concession, die ich gemacht habe, besteht darin, dafs ich die arsenige Säure beinahe in ihrem Aequivalent doppelt-kohlensaures Natrons auflöse. Ich vermeide dadurch Ueberschuls von Alkali, und wenn solches doch vorhanden wäre, es doch im möglichst wirkungslosen Zustande vorhanden sein müiste.

Meine Solutio mineralis Fowleri gab mil Silbernitrat den rein gelben Niederschlag, und mit Magnesiamixtur keine Spur von arseniksaurem Tripelsalze.

\title{
Ueber die schleimsauren Salze der Alkalien;
}

von S. W. Johnson aus New-York.

Man kannte bisher nur zwei Verbindungen der Schleimsäure, die für die zweibasische Nalur dieser Säure sprachen : das saure schleinsaure Kali, von-Hagen, und das saure sehleimsaure Aethyloxyd, von Malaguti dargestellt und beschrieben. 
Ich habe gefunden, dafs, wie Kali, so auch Natron und Ammoniak saure schleimsaure Salze von bestimmter Zusammensetzung bilden.

Saures schleimsaures Kali. - Aetzkali wurde mit Schteimsäure gesättigt und dann eine der zum Sältigen verbrauchten gleich grofse Quantitä von Schleimsäure hinzugefügt. Man erhät ein in feinen, farblosen Nadeln krystallisirtes Salz. Seine Zusammensetzung, luftrocken und bei $100^{\circ}$ getrocknet, wird durch die Formel KO, HO, $\overline{M_{u}}+2$ aq. ausgedriackl.

$0,2926 \mathrm{Grm}$. Substanz gaben $0,0961 \mathrm{KO}^{\mathrm{S} \mathrm{SO}^{3}}$.

$\begin{array}{cccc} & \text { Berechnet } & \text { Hagen } & \text { Johnson } \\ \text { KO } & 17,73 & 17,40 & 17,77 .\end{array}$

Saures schleimsaures Natron wurde ebenso wie das Kalisalz dargestellt. Es krystallisirt in farblosen glänzenden Prismen, welche an der Luft undurchsichtig werden durch Verlust von Krystallwasser. Das Salz wurde durch Pressen zwischen Papier rasch gelrocknet; es gab bei der Analyse folgende Zahlen :

1) 0,1366 Grm. Substanz verloren bei $100^{\circ} 0,0300 \mathrm{HO}$.

2) $0,2195 \mathrm{Grm}$. wasserfreje Substanz gaben 0,0658 $\mathrm{NaO}, \mathrm{SO}^{3}$.

3) $04700 \mathrm{Grm}$. Substanz verloren 0,1000 Wasser.

4) 0,4657 Grm. Substanz verloren 0,0984 Wasser.

5) 0,2385 Grm. wasserfreie Substanz gaben 0,0533 $\mathrm{NaO}, \mathrm{CO}^{2}$.

\begin{tabular}{crrrrrr} 
& & Berechnet & \multicolumn{3}{c}{ Gefunden } \\
NaO & 31 & 10,51 & & 10,76 & - & 10,75 \\
HO & 9 & 3,05 & & - & - & - \\
Mu & 192 & 65,08 & & - & - & - \\
7 aq. & 63 & 21,36 & & 21,96 & 21,27 & 21,22
\end{tabular}

Saures schleimsaures Ammoniak. - Wenn man eine Lösung von neutralem schleimsaurem Ammoniak bei böherer 
Temperatur ahdampft, so erhält man bekanntlich ein Salz von saurer Reaction. Es geht hier ein Theil des neutralen Salzes durch Verlust von Ammoniak in saures Salz über. Dieses saure Salz wird rein erhalten, wie die betreffenden Salze von Kali und Natron. Aus der heifsen Lösung scheidet es sich in farblosen Nadeln ab. Wenn man das Salz in grölseren Quanlitäten darstellt, so erhält man dünne, prismatische Kryslalle von einigen Linien Länge und Breite, die gemessen werden können. Diese Krystalle verlieren bei $100^{\circ}$ Wasser. Der Ammoniakgehalt derselben wurde durch Austreiben des Ammoniaks miltelst fixen Alkalis bestimmt.

Das entweichende Ammoniak wurde in einer bestimmten Quantität titrirter Schwefelsäure aufgefangen, und in dieser Flüssigkeit nach beendigtem Versuch der Gehalt an freier Säure durch Titriren bestimmt.

Bei den folgenden Versuchen wurde zuerst das Wasser in über Schwefelsäure getrocknetem Salz bei $100^{\circ}$ bestimmt; dann in diesem trockenem Salz das Ammoniak auf die eben beschriebene Methode erhalten.

0,8758 Grm. Substanz verloren 0,0640 HO.

1) 1,0709 Grm. Substanz gaben $0,1209 \mathrm{NH}^{4} 0$.

2) $0,9277, \quad \Rightarrow 0,1044 \%$

3) $0,8758, \quad, 0,0904 \%$

Es berechnet sich daraus die Formel : $\mathrm{NH}^{4} \mathrm{O}, \mathrm{HO}, \mathrm{Mu}+2$ aq.

Berechnet

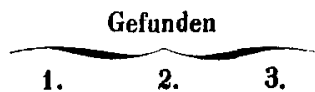

\begin{tabular}{rrrccc}
$\mathrm{NH}^{4} \mathrm{O}$ & 26 & 10,61 & 10,51 & 10,48 & 10,50 \\
$\mathrm{HO}$ & 9 & 3,67 & - & - & - \\
$\mathrm{Mu}$ & 192 & 78,37 & - & - & - \\
2 aq. & 18 & 7,35 & 7,34 & - & 7,30 \\
\hline \multicolumn{7}{c}{245} & $100,00$. & & &
\end{tabular}


Das saure schleimsaure Ammoniak isl in Wasser viel löslicher, als das neutrale Salz, ebenso auch die Salze des Kalis und Natrons.

Bei einer Bereitung des sauren schleimsauren Ammoniaks in grofsem Mafsstabe, welche ausgeführt wurde, um Material für das Studium der Zersetzungsproducte dieses Salzes zu gewinnen, wurden Krystalle von eigenthümlicher, merkwürdiger Zusammensetzung erhalten.

Ein Theil von der ersten Krystallisation, aus einer Kruste mit aufgewachsenen krystallen bestehend, gab, im Ganzen analysirt, folgende Zahlen :

1) $0,7111 \mathrm{Grm}$. Salz, über $\mathrm{SO}^{\text {s }}$ getrocknet, verloren 0,0297 Ho bei $100^{\circ}$.

2) $0,6712 \mathrm{Grm}$. ebenso getrocknetes Salz gaben 0,0446 $\mathrm{NH}^{4} \mathrm{O}$.

3) 0,6297 Grm. Salz gaben $0,0436 \mathrm{NH}^{4} \mathrm{O}$.

$\begin{array}{lll}\text { Wasser } & 4,17 & - \\ \mathrm{NH}^{4} \mathrm{O} & 6,65 & 6,92 .\end{array}$

Es war hier offenbar Schleimsäure beigemischt. Es wurden daher von den aufgewachsenen Krystallen einzelne vollkommen durchsichtige ausgewählt und diese analysirt.

Es wurde gefunden :

1) $0,9191 \mathrm{Grm}$. über $\mathrm{SO}^{3}$ getrocknetes $\mathrm{Salz}$ gaben bei $10000,0437 \mathrm{HO}$.

2) $0,8659 \mathrm{Grm}$. bei $100^{\circ}$ getrocknetes Salz gaben $0,1276 \mathrm{NH}^{4} \mathrm{O}$.

$$
\begin{array}{lr}
\text { Wasser } & 4,75 \\
\mathrm{NH}^{4} \mathrm{O} & 14,23 .
\end{array}
$$

Die Natur dieser Krystalle wird durch folgende Betrachtung klar :

4,75 Grm. Wasser bedürfen 6,85 Ammoniumoxyd, 53,09 Schleimsäure und 2,52 basisches Wasser, um saures schleimsaures Ammoniak zu bilden. Es blieben übrig in 100 Theilen : 
7,38 $\mathrm{NH}^{4} \mathrm{O}$ und 27,86 Schleimsäure.

7,38 $\mathrm{NH}^{4} \mathrm{O}$ bilden aber mit

27,24 Schleimsäure das neutrale Salz.

Die Zusammensetzung dieser Krystalle wird durch nachfolgendes Schema veranschaulicht :

\begin{tabular}{rl}
$\mathrm{NH}^{4} \mathrm{O}, \mathrm{HO}, \mathrm{Mu}+2$ aq. $.64,76$ & $=\left\{\begin{array}{rr}\mathrm{NH}^{4} \mathrm{O} & 6,85 \\
\mathrm{HO} & 2,52 \\
\mathrm{Mu} & 50,64 \\
2 \mathrm{aq.} & 4,75\end{array}\right.$ \\
$2\left(\mathrm{NH}^{4} \mathrm{O}\right), \mathrm{Mu} \quad . \quad . \quad . \quad 34,62$ & $=\left\{\begin{array}{rr}\mathrm{NH}^{4} \mathrm{O} & \mathbf{7 , 3 8} \\
\mathrm{Mu} & 27,24\end{array}\right.$ \\
\hline 99,38 & $99,38$.
\end{tabular}

Ein anderer Theil Krystalle von derselben Darstellung wurde mit gröfster Sorgfalt ausgesucht.

1) 0,3975 Grm. über $\mathrm{SO}^{3}$ getrocknetes $\mathrm{Salz}$ gaben bei $110^{\circ} 0,0251$ Grm. Wasser.

2) $0,3708 \mathrm{Grm}$. bei $110^{\circ}$ getrocknetes Salz gaben $0,0467 \mathrm{NH}^{4} 0$.

$$
\begin{array}{lr}
\text { Wasser } & 6,31 \\
\mathrm{NH}^{4} \mathrm{O} & 11,85 .
\end{array}
$$

6,31 Grm. Wasser bedürfen 9,10 Ammoniumoxyd, 67,28 Schleimsäure und 3,15 basisches Wasser, um saures Salz zu bilden. Es bleiben übrig 2,75 Ammoniumoxya, welche sich nit 10,15 Schleimsäure zu neutralem Salz verbinden.

Um 100 auszumachen, fehlen 1,26 ; es wurde offenbar etwas zu wenig Ammoniak gefunden, welcher Fehler sich für die Schleimsäure bedeutend multiplicirt.

$$
\begin{aligned}
& \mathrm{NH}^{4} \mathrm{O}, \mathrm{HO}, \mathrm{Mu}+2 \text { aq. . } .85,84=\left\{\begin{array}{cc}
\mathrm{NH}^{4} 0 & 9,10 \\
\mathrm{HO} & 3,15 \\
\mathrm{Mu} & 67,28 \\
2 \text { aq. } & 6,31
\end{array}\right.
\end{aligned}
$$

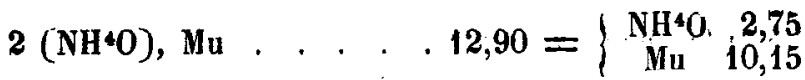

$$
\begin{aligned}
& 98,7498,74 \text {. }
\end{aligned}
$$


Die einzelnen Krystalle zu diesen Analysen waren nicht durch Messung bestimmt, aber sie waren dem Ansehen nach alle von gleicher Form.

Die Thatsache, dafs bei der Bereitung des neutralen Salzes Krystalle erhalten werden, die sauer reagiren, spricht dafür, dafs das neutrale Salz mit dem sauren in unbestimmten Verhältnissen zusammenkrystallisirt.

Obige Analysen bestätigen dlefs vollkommen, die beiden Salze scheinen in der That isomorph zu sein, aber nur in krystallographischem, nicht in chemischem Sinn, denn sonst mülste man annehmen, dals Ammoniumoxyd isomorph wäre mit $\mathrm{HO}+2$ aq., oder $\mathrm{NH}^{4}$ mit $\mathrm{H}+2$ aq., was natürlich undenkbar ist. Die Annahme, dafs beide Salze isomorph sind, mufs durch Messungen bewiesen werden.

Die Analyse Nr. 3, von saurem schleimsaurem Ammoniak, wurde von Krystallen des reinen sauren Salzes gemacht, welche weder in Grölse noch in Form von den Krystallen des Doppelsalzes dem Ansehen nach verschieden waren.' Sie wurden durch langsame Verdampfung der Multerlauge erhalten.

Die Bildung von neutralem Salz in der Lösung von saurem wird offenbar bedingt durch die Abscheidung von Schleimsäure, währ'end die Bildung des sauren Salzes in der Lösung des neutralen durch die Flüchtigkeit des Ammoniaks hervorgebracht wird.

In heiden Fällen wird das Bastardsalz gebildet.

Bei der Darstellung dieser Salze wurde wahrscheinlich Paraschleimsäure gebildet, weil man die Schleimsäure mit viel Wasser und Ammoniak längere Zeil kochen muls, um sie aufzulösen, und weil diese verdünnte Lösung dann weit eingedampft werden mufs, ehe sie krystallisirt.

Bekanntlich scheidel eine heilse Lösung von Paraschleimsäure heim Erkalten gewöhnliche Schleimsäure ab. 
Die Zerselzungsproducte des sauren schleimsauren Ammoniak's scheinen ziemlich dieselben zu sein, wie die von $\mathrm{Ma-}$ laguli aus dem neutralen Salze erhaltenen, gröfstentheils kohlensaures Ammoniak, Bipyromucamid und Gase.

Daneben erhielt ich eine kleine Quantität eines klaren, gelben Oeles, von brenzlichem Geruch, in Wasser löslich, was, wie es scheint, einen unter $100^{\circ}$ liegenden Siedepunkt hat, da es, mit Wasser destillirt, in den ersten Theilen des Destillates enthalten ist. An der Luft bräurt sich dieser Körper rasch. Es wurde bis jetzt nicht näher untersucht Ich werde sobald wie möglich die Untersuchung dieses Gegenstandes wieder aufnehmen.

\section{Ueber die Hypogäsäure, eine neue Fettsäure im Erdnufsöl ;}

von Dr. A. Gö/smann und H. Scheven.

In einer früheren Abhandlung*) hal der eine von uns bereits mitgetheilt, dafs in dem Erdnufsöl, dem Oele, welches aus den Früchten der Arachis hypogaea gewonnen wird, eine neue Fettsäure, die Arachinsäure $=\mathrm{C}^{40} \mathrm{H}^{40} \mathrm{O}^{4}$, enthalten ist. Die nun gemeinschaftlich von uns fortgesetzte Untersuchung dieses Oeles hat uns auf die Auffindung noch einer neuen, d. s. g. Oelsäure - Reihe $=\mathrm{C}^{\mathrm{n}} \mathbb{H}^{\mathrm{n}-2} \mathrm{O}^{4}$ angehörenden Säure geführt. Diese neue Säure, die wir Hypogäsäure nennen wollen, hat die Zusammensetzung $\mathrm{C}^{52} \mathrm{H}^{\circ 0} \mathrm{O}^{4}=\mathbf{H}$ $+\mathrm{C}^{33} \mathrm{H}^{20} \mathrm{O}^{3}$. Die Gewinnung und Reinigung, sowie die Nachweisung ihrer Eigenthümlichkeit, wollen wir in dem Fol-

*) Diese Annalen LXXXIX, 1. 